AlaA Space 2001 - Conference and Expesition, Albuquerque, NM, Aug. 28-30, 2001

\section{A01-39846}

AIAA 2001-4610

\title{
INTERFEROMETRIC IMAGING CONCEPTS WITH REDUCED FORMATION-KEEPING CONSTRAINTS*
}

\author{
David C. Hyland \\ Department of Aerospace Engineering \\ The University of Michigan
}

\begin{abstract}
$\underline{\text { Abstract }}$
The Origins program and corresponding next generation observational facilities seek orderof-magnitude advances in optical angular resolution via long baseline interferometry. We consider systems involving a collection of separate free-flying, light collecting spacecraft, or optical processing spacecraft, where all the spacecraft are required to fly in precise formation in order to form the image of a very distant object.

In the investigation discussed here, we consider the reduction of system complexity by the use of novel imaging system architectures that relieve constraints on formation keeping. Considering the system configuration, as currently conceived for both starlight nulling and imaging, there are numerous inter-spacecraft positioning and system geometry constraints that must be satisfied to assure successful image formation. We discuss each of these constraints, in turn, and attempt to alleviate the constraint by means of various alternative approaches, including enhanced algorithms, advanced signal processing, nonlinear optics, and novel optoelectronic systems which have the potential to alleviate or even eliminate the constraint.
\end{abstract}

\section{Introduction}

The goals of the Origins program and corresponding next generation observational facilities entail order-of-magnitude advances in optical angular resolution. Increased angular resolution is sought from interferometric systems with large baselines between the widely separated apertures of interferometers. We address concepts such as $\mathrm{TPF}^{1}$, involving a collection of separate free-flying, light collecting spacecraft, each one carrying a relatively modest-sized optical or infrared sensor, where the distance between spacecraft can be many kilometers. Besides the light collecting spacecraft, the conventional system concept includes precisely aligned spacecraft that actually combine the collector beams, perform starlight nulling and carry out the interferometric measurements needed for the synthesis of highresolution images. Within these concepts, all the spacecraft are required to fly in precise formation in order to form the image of a very distant object. This must be accomplished without frequent human intervention.

The present investigation considers potential reductions in cost and complexity by the use of novel imaging system architectures that relieve constraints on formation keeping, and formation flying control formulations that fully account for the system's image forming performance. In this paper we review the various formation-keeping geometric constraints entailed in current system architectures and discuss various avenues to alleviating or eliminating them.

Figure 1 shows a general architecture, following current concepts, for an exo-solar planet imager, including both nulling and imaging components. The precision requirements on

\footnotetext{
* Copyright $\odot 2001$ by the American Institute of Aeronautics and Astronautics, Inc. All rights reserved.

- Professor, Department of Aerospace Engineering, Member
} 
formation keeping are indicated in the boxes. All the positioning requirements shown have to be maintained within a fraction of a wavelength. The very precise $(<1$ nanometer) positional and attitude adjustments would be made via active optics within the several spacecraft, while the "coarse" adjustments are made through the spacecraft attitude control effectors. Nevertheless, the "coarse" attitude control still requires high precision maneuver control -- typically $1 \mathrm{~cm}$ accuracy over 1 to $10^{3}$ kilometers or more baseline. Hence the constraints shown in the Figure are quite severe. In the following sections, we describe each formation-keeping constraint in turn and describe a number of technological advances that could and alleviate or eliminate them

\section{Constraint 1}

The notion that light collection for interferometric imaging must occur over a single ("u-v") plane probably arises from the widely quoted van Cittert-Zernike result, which is derived from an approximate version of the HuygensFresnel principle and specialized to a planar observation surface ${ }^{2}$. However, a generalization of the popular van Cittert-Zernike result can readily derived $^{2,3}$ which gives a readily computed integral expression for the image plane intensity given interferometrically measured values of the mutual intensity function on a general, non-planar observation surface or even a three-dimensional volume. This result is illustrated in Figure 2 . Implications are: (1) Light collection for imaging need not be constrained to occur on a plane and (2) The imaging algorithm allows relative position knowledge (from metrology) to be combined with processing to compute image data even when the nulling pods are at different ranges from the target of observation.

The result shown in Figure 2 gives rise to a computationally efficient algorithm for computing the image so far attained from past and present data. The algorithm requires only the mutual intensity measurement data together with accurate knowledge of the relative positions of the light collecting apertures. Control of the relative positions of the nulling pods to conform to some $a$ priori geometric constraint is not needed and light collector spacecraft may be distributed over some three-dimensional volume.

Thus above result significantly relieves the relative positioning requirements on the spacecraft constellation. Essentially, we use positional knowledge (from metrology) and signal processing to replace relative position control. Since, in any case, a metrology system of the same accuracy is needed anyway, this modified scheme reduces cost and adds system flexibility.

\section{Constraints $2 . \mathrm{a}$ and $2 . \mathrm{b}$}

To alleviate the precise alignment requirements for space segments within a nulling pod, one might seek suitable optical delay devices that could be hosted within the individual light collector spacecraft. Using relative position data from metrology, these devices could be used to actively correct for positioning errors, even adaptively steering the null of the pod without much accuracy in inter-spacecraft positioning control. With the estimates given in Figure 1 (with $\mathrm{D}_{\mathrm{N}} \approx 100 \mathrm{~m}$ ) even optical delay devices such as are contemplated for ST-3 (with $20 \mathrm{~m}$ path length) would greatly relieve the formation-keeping constraints. It is desirable to develop delay devices of similar path length that are compact and variable. Besides piezo-driven mirror arrangements, one might consider the use of optoelectronic delay devices. This is another instance of using positional knowledge and optoelectronics to obtain path length control without directly positioning the spacecraft.

Variable delay devices mounted within the first-level combiners would similarly relieve the need to align them relative to their pod collectors. Thus progress in relieving constraint 2.a would help here as well. Further, if sufficiently large path length delay devices can be developed, it might be possible to eliminate the first-level combiner as a distinct spacecraft. Analogous to ST3 , light from all of the collectors of a pod would be propagated to one of the collectors and the delays would be used to readjust path length prior to combining the beams.

\section{Constraint 3 - Phasing Beacon Approach}

The output beams of the nulling pods are the initial inputs to the interferometric measurements. To perform these measurements, even without constraints on the relative locations of nulling pods, the second-level combiner spacecraft must still fly in precise alignment with the pair of nulling pods being used. Referring to the estimates in Figure 1, the typical baseline among separate pods could be on the order of $10^{7} \mathrm{~m}$, so the use of delay devices to alleviate the requirements on second-level combiner positioning or to eliminate 
the need for such combiners is out of the question. At the same time, with $\mathrm{D} \approx 10^{7} \mathrm{~m}$, the conventional approach by which the output beams of two nulling pods are propagated to the second-level combiner, where the beams are physically interfered faces severe challenges because of this propagation distance. An alternative, which we consider, may be called the "Phasing Beacon" approach. Here, as illustrated in Figure 3, a laser beacon of frequency $\omega_{0}$ is propagated from one first-level combiner to the others. Small stroke delay devices in each spacecraft are used together with metrology knowledge to equalize the phase of the beacon before it is interfered with the collected beam from each pod combiner. Each pod beam and the phased-up beacon are interfered in an optical demodulator consisting of conventional components and detectors. Each demodulator might take the form shown in Figure 4, where the input is the frequency content of the collected light within the band $\left[\omega_{0}-\Delta \omega, \omega_{0}+\Delta \omega\right]$. The outputs are the in-phase and quadrature components of this signal. Figure 4 shows the set up for the in-phase component while the quadrature component is handled analogously.

The output of the device in Figure 4 is the output of the optical detectors with $(\mathrm{A} / 8) \mathrm{U}_{\Delta \lambda} \cos \omega t$ as input. Let us consider the nature of the spectral content of the output signal. We may suppose that the detector's frequency response is that of some low-pass filter, $\mathrm{B}_{\mathrm{D}}(\omega)$ with a frequency cut-off at $\omega_{D}\left(\omega_{D}=\Delta \omega\right)$, that is: $B_{D}(\omega)=0$ for $|\omega| \geqq \omega_{D}$. We can also think of $U_{\Delta \lambda}$ as the output of the filter $\mathrm{B}_{\mathrm{D}}(\omega+\varpi)+\mathrm{B}_{\mathrm{D}}(\omega-\varpi)$. With these assumptions and denoting the Fourier transform of $U$ by $F_{U}(\omega)$, we have:

$$
\begin{aligned}
U_{\Delta L} & =\frac{1}{2 \pi} \int d \omega e^{i \omega t}\left[B_{D}(\omega-\varpi) F_{U}(\omega)+B_{D}(\omega+\varpi) F_{U}(\omega)\right] \\
& =U_{C} \cos \varpi t+U_{S} \sin \varpi t
\end{aligned}
$$

where:

$$
\begin{aligned}
& U_{C}=\frac{1}{2 \pi} \int d \omega e^{i \varpi t}\left[B_{D}(\omega)\left(F_{U}(\omega+\varpi)+F_{U}(\omega-\varpi)\right)\right] \\
& U_{S}=\frac{i}{2 \pi} \int d \omega e^{i \varpi t}\left[B_{D}(\omega)\left(F_{U}(\omega+\varpi)-F_{U}(\omega-\varpi)\right)\right]
\end{aligned}
$$

On the other hand, the Fourier transform of the output of the device in Figure 4 is:

$$
\begin{aligned}
& =B_{D}(\omega) \int d t e^{-i \varpi t} \cos \varpi t U(t) \\
& =B_{D}\left[F_{U}(\omega-\varpi)+F_{U}(\omega+\varpi)\right] \\
& =F_{U_{C}}(\omega)
\end{aligned}
$$

Thus the output of the device is $\mathrm{U}_{\mathrm{C}}$ and a similar device produces $U_{S}$. The above expressions for $U_{C}$ and $U_{S}$ show that the frequency content of these quantities encompasses only $[-\Delta \omega,+\Delta \omega]$.

In other words, the variation of an electromagnetic field variable associated with radiation in this band, $U(t)$, has the form: $U(t)=U_{c}$ $(\mathrm{t}) \cos \omega \mathrm{t}+\mathrm{U}_{\mathrm{s}}(\mathrm{t}) \sin \varpi \mathrm{t}$. The demodulator both generates $U(t)$ and extracts $U_{c}(t)$ and $U_{s}(t)$. While these coefficients are still very rapidly varying, they are of much lower frequency than the "carrier frequency", $\varpi$, and can be digitally recorded. Through a communications link, these signals from two pod combiners are sent to a central location (e.g. one of the combiners) where the mutual intensity function corresponding to the two pod locations is digitally computed according to:

$$
\mathrm{J}\left(\mathrm{P}_{1}, \mathrm{P}_{2}\right)=<\mathrm{U}_{\mathrm{c} 1} \mathrm{U}_{\mathrm{c} 2}+\mathrm{U}_{\mathrm{s} 1} \mathrm{U}_{\mathrm{s} 2}>
$$

Here $U_{c 1}, U_{s 1}$ and $U_{c 2}, U_{s 2}$ are the modulation coefficients from pods 1 and 2, respectively and $\langle\ldots\rangle$ denotes time average. This measurement scheme is accomplished for several frequency bands simultaneously to cover the frequency region of observational interest.

Figure 5 illustrates the inherent trade off between the width of the individual frequency bands, as characterized by the spectral resolution, $\mathrm{R}=\lambda / \Delta \lambda$, and the maximum frequency of the demodulated signal. Clearly if we are willing to utilize sufficiently many wavelength channels for a given total wavelength range (that is, we make $R$ large enough), the upper frequency range of the demodulated signal can be made as small as desired. The constraint is that this frequency must be below the frequency response of the detectors. The indicated limit of IR detector response is based on relatively established technology in which a low-temperature-grown GaAs detector with a metal-semiconductor-metal structure achieves a bandwidth of $375 \mathrm{GHz}^{4}$. As Figure 5 shows, in the IR imaging regime, with an $R$ of several hundred, the optical signal can be down-converted to the EHF band. These values entail high-speed processing and advanced electronics but are well within the realm of possibility.

Note that the phasing beacon approach does not propagate collected light beams between spacecraft, but rather, it involves propagation of coherent laser beams, a much more tractable task, especially over very long paths. Secondly, since the mutual intensity is formed computationally rather than by physical interference of collected beams, the second level collector spacecraft can be 
eliminated from the architecture. Further development of this sort of down-conversion technology could revolutionize long baseline interferometry.

\section{Constraint 3 - Electric Field Reconstruction}

The ultimate in achieving system modularity and in avoiding the need to propagate collected light beams over long baselines and physically interfere them to perform the needed interferometric measurements would be a scheme in which, at every light collecting spacecraft, the electric field is sampled over a train of successive pulses and the Fourier transform of the electric field is then determined. In effect, the electric field is determined at each location, transformed into digital data and the data sent to some central location (perhaps one of the spacecraft) where it is used to calculate the mutual intensity at all pairs of locations. Such direct electric field reconstruction (or, "E-field reconstruction") techniques have been developed, originally for applications involving quantum control ${ }^{5-10}$. It is only recently in this investigation that we have explored their use for the optical processing required for long baseline interferometric imaging. Two categories of methods may be discerned: Self-referencing and linear methods. As the term implies, selfreferencing methods are capable of reconstructing an electric field pulse without the use of an externally supplied reference signal. One such method $^{5}$ is shown in Figure 6. An experimentally mature method is discussed in references 6 and 7. While these methods are self-contained, the data needed for the Fourier transform phase reconstruction is proportional to a high power (fourth and even sixth) of the light intensity so that the methods are not suitable for low light intensity. On the other hand, linear methods, such as Fourier Transform Spectral Interferometry $\left(\mathrm{FTSI}^{8-10}\right.$ ), use data proportional to the first power of the intensity and are thus effective at low light levels. However, these methods require the use of an externally supplied reference signal.

To address the above complementary strengths and limitations, we are investigating a hybrid method. This proceeds by first synthesizing a moderate intensity beam with significant spectral content in the frequency band of the light to be analyzed, then fully characterizing this beam with a self-referencing method. We then use this reference signal and FTSI to determine the magnitude of the transform of the unknown beam and the difference of the phase of the unknown beam and the reference beam. Since we have data on the phase of the transform of the reference beam by means of the self-referencing method, we can determine the phase of the unknown beam. By this means, pulses of low intensity light collected at each spacecraft location can be transformed into digital data on the frequency or time domain behavior. This data can be saved, sent to other locations or used within the imaging constellation to compute high-resolution images.

\section{Summary of System Architecture Ramifications}

To summarize, we see that the substitution of optoelectronics, optical computing and signal processing for reliance on traditional optical devices has the potential to greatly mitigate or remove the four constraints discussed above and radically simplify the system architecture. The ultimate simplification is one in which a widely dispersed, loosely organized "swarm" of functionally identical spacecraft-borne optics would use a precise metrology system and largescale, parallel signal processing to perform interferometric imaging without the requirement of precise inter-spacecraft positioning and alignment control. The reduction of system control requirements introduces a considerable improvement of flexibility and reduction of cost.

It should be noted that it is still necessary to strategically command segment motions to sweep out the correct regions over the observation region so as to efficiently construct the mutual coherence on the image plane. In fact, most of the challenges and motivations of much of the past work on formation flying control (see for example, references 11-13) retain their relevance for this alternative system concept. The main difference relative to conventional system concepts is that a set of formation-keeping constraints are removed, the set of feasible control actions is larger and, in particular, there is wider scope for the use of passive formation forming strategies.

It is also clear that imaging maneuvers involving distributed space systems require the ability to devise and execute, without need of ground-control intervention, whatever formation maneuvers are needed to produce an image, of acceptable quality, of the designated object. Current work in formation flying control has focused on control optimization to execute prescribed maneuvers with minimum time or fuel. The prescribed maneuvers are synthesized by determining an open loop control policy that optimizes some imaging figure of merit, such as the point spread function (PSF), or Modulation 
Transfer Function that is calculated according to $a$ priori models rather than measured in situ and used in truly closed-loop control. To achieve truly autonomous, closed-loop capability, work is need to incorporate into the formation flying control formulation a "figure of merit" that also includes imaging performance of the system i.e. the quality of the image so-far attainable through the course of the observations taken over the current formation flying maneuver.

To compute optimal closed-loop formation maneuvering policies, one could use such standard characterizations of image quality as the PSF - or, more precisely, the root-mean-square error between the calculated PSF and a postulated ideal PSF. As an alternative, one might consider image quality measures that can be estimated online by the system. Optimization of an integrated figure of merit that includes this measurable estimate of image quality would enable more "selfreliant" formation flying control that assures the system will carry out maneuvers to acquire an image of acceptable quality as rapidly as possible and without frequent re-direction from ground control.

Composition of this figure of merit entails two steps: (1) Transformation of interferometric measurements of mutual intensity on the observation plane (or surface) to the mutual intensity on the image plane (using an algorithm derived from the result shown in Figure 2) and (2) computation, from image plane intensity, of a measure of image definition/completeness. Using existing capabilities in item (1), work in item (2) might address measures of image quality that can cope with unknown image content e.g. one may be observing a previously unknown terrestrial-sized planet yet one needs assurance that the image will be "sharp". Various measures of contrast will be addressed, in particular, image entropy in the information theoretic sense or, more generally, conditional entropy as well as a variety of imaging figures of merit that characterize image completeness, or address the identification of image features such as points, lines or edges. Such figures of merit are available for standard images obtained from cameras and can be expressed in terms of statistical measures that are directly computable from the image data. Similar figures of merit can be identified and computed for images obtained from interferometric imaging approaches. The resulting integrated measure of image quality will be a complex function of the formation flying patterns and maneuvers - reflecting, for example, the set of points swept out by the light collecting elements of the constellation in the observation plane. This figure of merit also provides the basis for the extensive work that is needed to bring into play notions of pupil configuration optimization ${ }^{14}$ and image reconstruction techniques ${ }^{15}$.

Further, there is need for a very high degree of autonomy in the decision/control system that plans and executes imaging maneuvers. Over the past two decades, control technology, including a wide spectrum of activities from algorithms to hardware, has made enormous strides in improving pointing and tracking precision and image quality for NASA and Air Force space systems. Although significant enhancements to acquisition, tracking and vibration suppression were demonstrated by a number of laboratory programs, it is clear as a part of the "lessons learned" from these laboratory demonstrations that widespread system application demands a further quantum jump in control technology. Specifically, the application of advanced control technology aboard future missions requires the development of self - reliant control systems. This implies a capability much more demanding than the now-familiar robust, multivariable control. Self-reliance implies a degree of in situ intelligence that enables a system to (1) perform successfully without constant human intervention, (2) devise, monitor and adapt its control policy, (3) work around faults/failures, recover control effectiveness, (4) monitor and report health and status and (5) perform maintenance and repair. In other words, the control system, considered to be hardware, processors and algorithms, should be able to "take care of itself" hence the term "self-reliant". The need for this is especially keen for NASA Origins program missions because the space assets will be deployed in highly remote locations with the expectation of complex mission operations despite very lengthy communications travel times. For such systems, the ability to self-reliantly monitor and revise control laws and to monitor health and status and diagnose problems are absolute necessities.

With the figure of merit that integrates imaging quality as described above, we might contemplate the closed-loop system illustrated in Figure 7. This is a multi-level, intelligent, knowledge-based control and decision system that has the required self-reliance attributes. In the process of image formation in response to a mission control request, the system would autonomously acquire interferometric data, use this to compute the best estimate of the image reconstructed so far, determine an image quality metric and use this metric to drive a dynamic programming algorithm for determining the most effective subsequent maneuvers to most rapidly 
improve the image quality. The process stops when the system has determined that image quality has achieved a required standard. The development of this kind of autonomous control/decision system for constellation control can build upon much previous experimental and theoretical work ${ }^{16}$. Finally, to utilize a free-flyer interferometer constellation most efficiently, we should be able to exploit the natural dynamics of the constellation so that the spacecraft sweep over a sufficiently rich portion of the observation surface to form a high quality image while expending minimum fuel and power. Thus, much effort, in the vein of reference 17 is needed in optimal orbit/constellation design.

\section{$\underline{\text { References }}$}

1. "Terrestrial Planet Finder", JPL Publication 993, May 1999, http:/tpf.jpl.nasa.gov/library/tpf_book/index.html.

2. M. Born and E. Wolf, Principles of Optics: Electromagnetic Theory of Propagation, Interference and Diffraction of Light, Macmillan Co. , New York, 1964.

3. D.C.Hyland, "The Inverse Huygens-Fresnel Principle and Its Implications for Interferometric Imaging", Journal of the Astronautical Sciences, to appear.

4. G. K. Chen, "Development of Detectors and Modulators for Multi-Hundred GigaHertz Operations (Optoelectronics)", Ph.D. Dissertation, The University of Michigan, 1991.

5. T. I. Kuznetsova and I.A. Walmsley, "Reconstruction of temporal signals from nonlinear optical measurements", Quantum Electronics, 28 (8), 728-732, 1998.

6. C. Iaconis, and I. A. Walmsley, "Spectral phase interferometry for direct electric-field reconstruction of ultrashort optical pulses", Opt. Soc. Am., Jan. 1998.

7. C. Iaconis, and I. A. Walmsley, "Selfreferencing spectral interferometry for measuring ultrashort optical pulses", IEEE Jour. of Quantum Electronics, Vol.35, No. 4, April, 1999.
8. L. Lepetit, G. Cheriaux, and M. Joffre, "Linear techniques of phase measurement by femtosecond spectral interferometry for applications in spectroscopy", J. Opt. Soc. Am. B, Vol.12, No. 12, December, 1995.

9. C. Dorrer, "Implementation of spectral phase interferometry for direct electric-field reconstruction with a simultaneously recorded reference interferometry", Optics Letters, Vol.24, No. 21, November, 1999.

10. C. Dorrer, N. Belabass, J.-P. Likforman, and $M$. Joffre, "Spectral resolution and sampling issues in Fourier-transform spectral interferometry", $J$. Opt. Soc. Am., Vol. 17, No. 10, October, 2000.

11. P. K. C. Wang and F. Y. Hadaegh, "Coordination and Control of Multiple Spacecraft Moving in Formation", Jour. Of the Astronautical Sciences, Vol. 44, No. 3, 1996.

12. P. K. C. Wang and F. Y. Hadaegh, "Optimal Formation-Reconfiguration for Multiple Spacecraft”, AIAA GN\&C Conf., August, 1998.

13. F. Y. Hadaegh, E. Miller and C. F. Lin, "Autonomous Spacecraft Guidance and Control", AIAA GN\&C Conf., July 1996.

14. L. M. Mugnier and G. Rousset, "Pupil configuration optimality criterion in synthetic aperture optics", in Spaceborne Interferometry II, Proc. SPIE, Vol. 2477, pp. 124-131, 1995.

15. G. Demoment, "Image reconstruction and restoration: Overview of common estimation structures and problems", IEEE Trans. On Acoustics, Speech, and Signal Processing, Vol. 37, No. 12, December, 1989.

16. K.K. Denoyer, D.C. Hyland, L.D. Davis and D.W. Miller, "MACE II: A Space Shuttle Experiment for Investigating Adaptive Control of Flexible Spacecraft," Paper No. AIAA-98-4319, AIAA Guidance, Navigation and Control Conference, Boston, MA, August 10-12, 1998.

17. D.J. Scheeres, and Y. Hou. 1998. "The Dynamics of Uncertainty: Measuring Orbit Knowledge and Quality", AIAA Paper 98-4559. Submitted to the Journal of Guidance, Control, and Dynamics, 1999. 


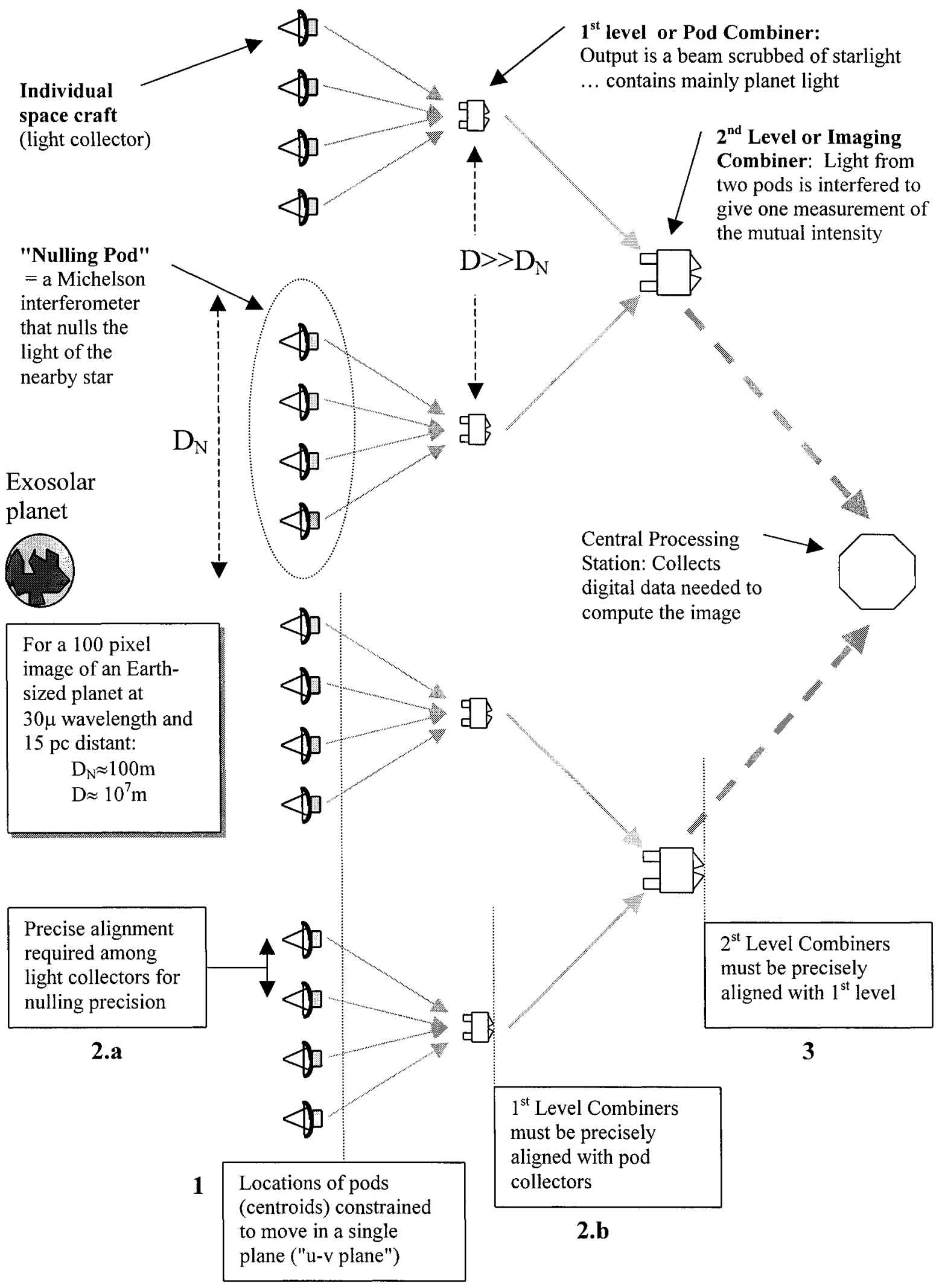

Figure 1: General planet imaging system architecture - Both nulling and imaging 


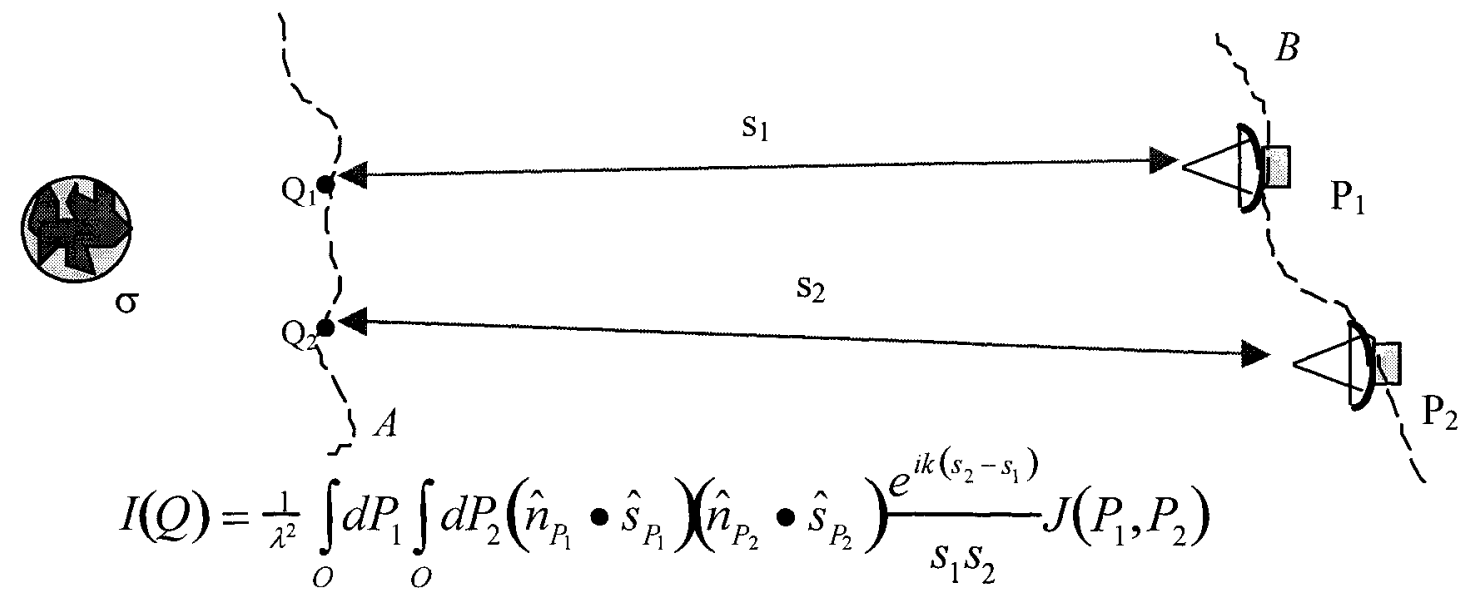

Figure 2: General Image synthesis result: Surfaces A and B need not be planes.

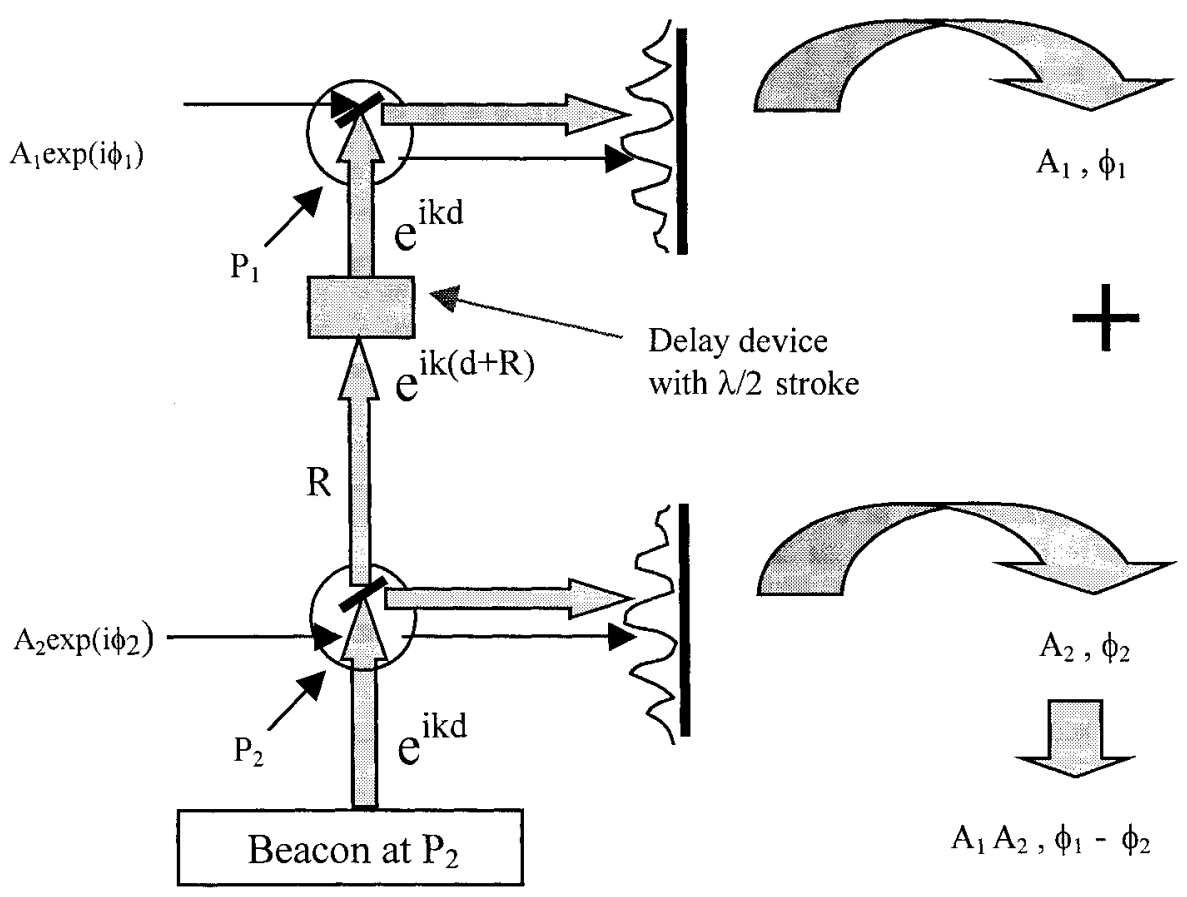

Figure 3: Phasing Beacon To Extract Mutual Intensity W/O Propagating Collected Light Among S/C 


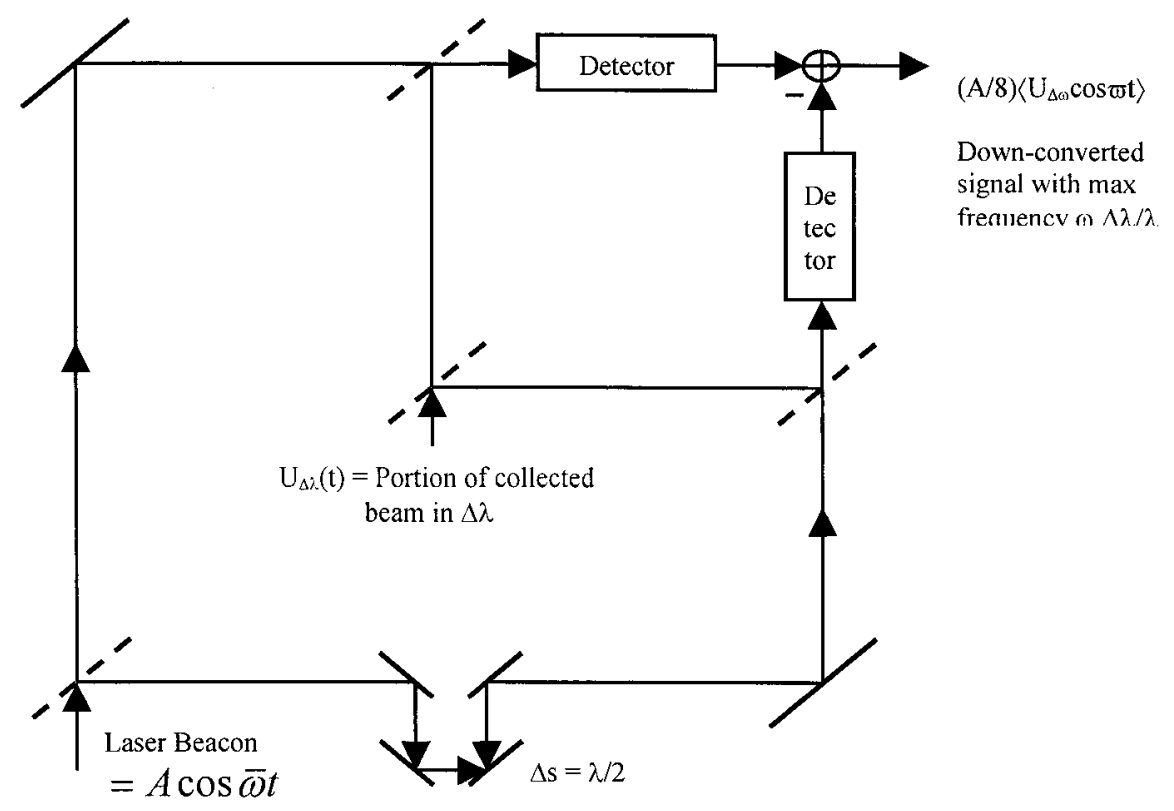

Figure 4: Optical demodulator component for the in-phase component.

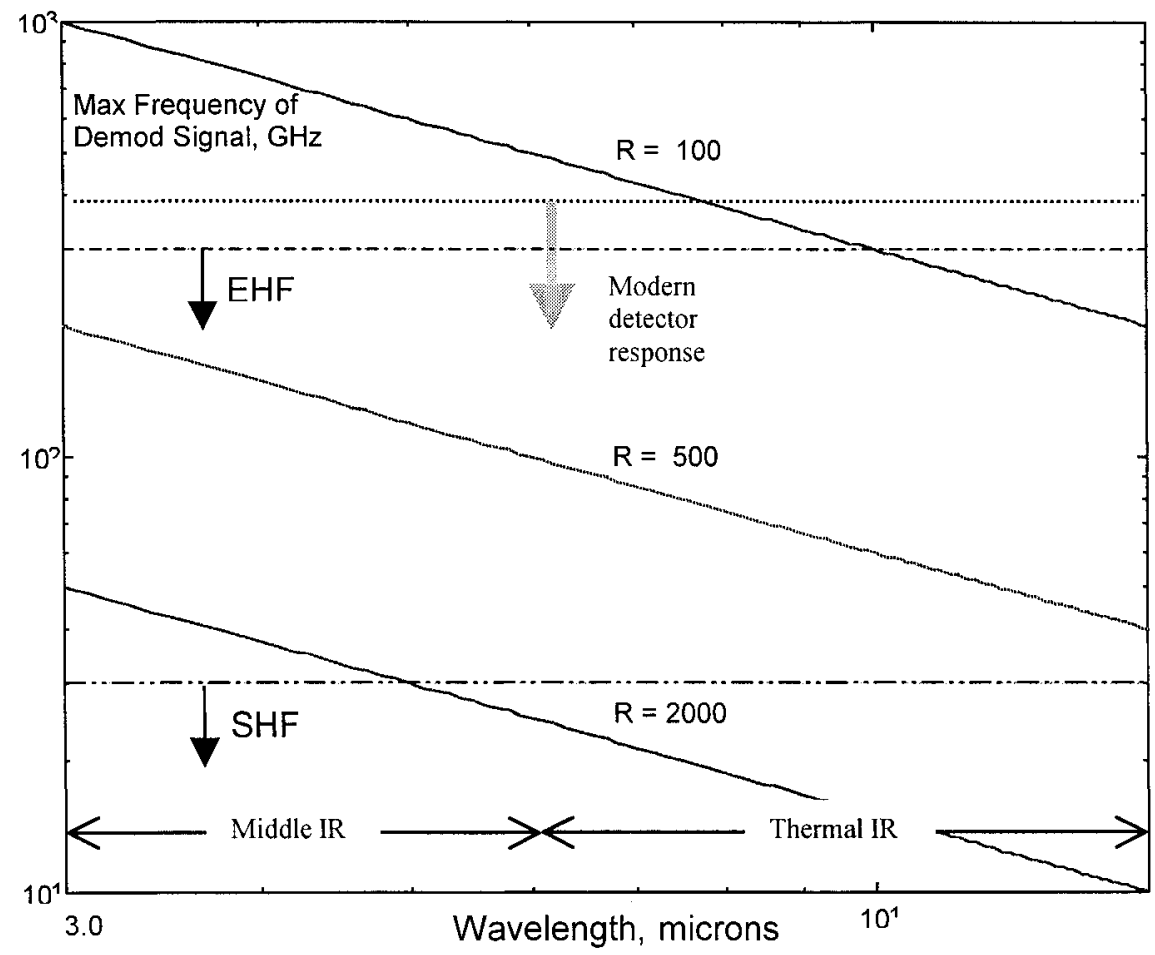

Figure 5: Maximum frequency of demodulated signal as a function of center-band wavelength for various spectral resolution value, $R$. 

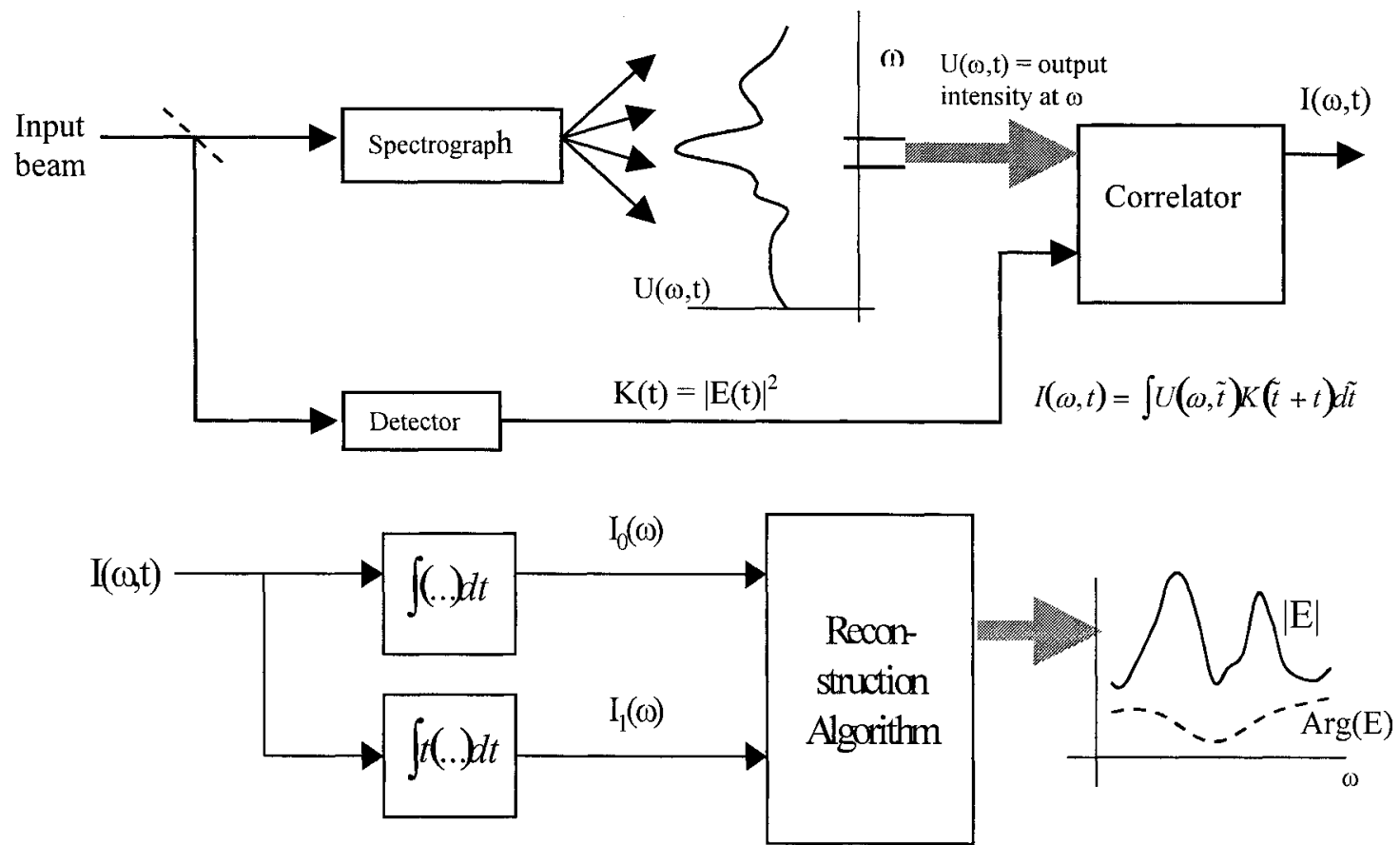

Figure 6: Reconstruction of the Electric Field - Correlation Method

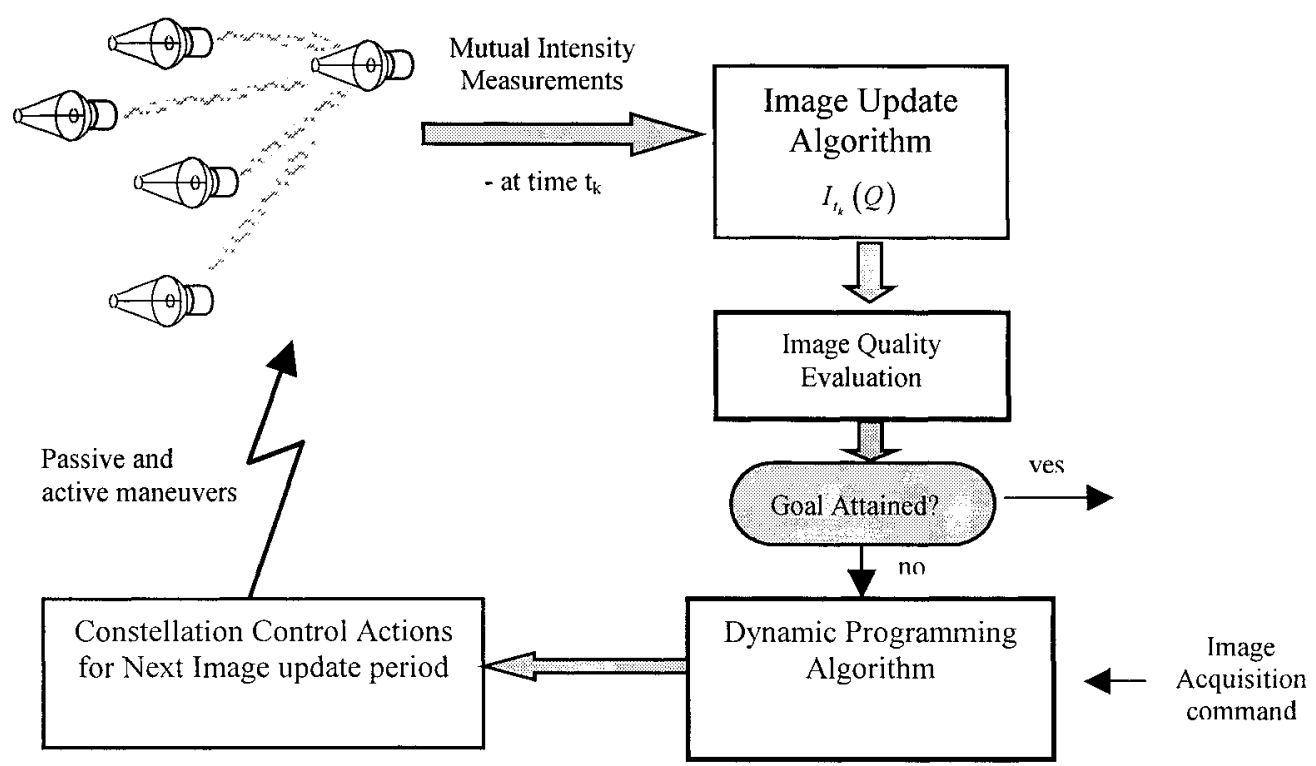

Figure 7: Closed-loop imaging constellation control 